\title{
Brain-Machine Interface Induced Morpho-Functional Remodeling of the Neural Motor System in Severe Chronic Stroke
}

\author{
Andrea Caria ${ }^{1,2,3}$ (D) Josué Luiz Dalboni da Rocha ${ }^{4} \cdot$ Giuseppe Gallitto $^{1} \cdot$ Niels Birbaumer $^{3} \cdot$ Ranganatha Sitaram $^{5,6,7}$. \\ Ander Ramos Murguialday ${ }^{3,8}$
}

Published online: 4 December 2019

(C) The American Society for Experimental NeuroTherapeutics, Inc. 2019

\begin{abstract}
Brain-machine interfaces (BMI) permit bypass motor system disruption by coupling contingent neuroelectric signals related to motor activity with prosthetic devices that enhance afferent and proprioceptive feedback to the somatosensory cortex. In this study, we investigated neural plasticity in the motor network of severely impaired chronic stroke patients after an EEG-BMIbased treatment reinforcing sensorimotor contingency of ipsilesional motor commands. Our structural connectivity analysis revealed decreased fractional anisotropy in the splenium and body of the corpus callosum, and in the contralesional hemisphere in the posterior limb of the internal capsule, the posterior thalamic radiation, and the superior corona radiata. Functional connectivity analysis showed decreased negative interhemispheric coupling between contralesional and ipsilesional sensorimotor regions, and decreased positive intrahemispheric coupling among contralesional sensorimotor regions. These findings indicate that BMI reinforcing ipsilesional brain activity and enhancing proprioceptive function of the affected hand elicits reorganization of contralesional and ipsilesional somatosensory and motor-assemblies as well as afferent and efferent connection-related motor circuits that support the partial re-establishment of the original neurophysiology of the motor system even in severe chronic stroke.
\end{abstract}

Key Words Brain-machine interface $\cdot$ stroke $\cdot$ DTI $\cdot$ functional connectivity $\cdot$ motor recovery

\section{Introduction}

Advances in the brain-machine interface (BMI) field have shown that human patients with severe neurological insults can artificially bypass the injury and re-animate paralyzed limbs through learned control of external devices [1-3].
Tetraplegic patients through implanted multielectrode array, recording spike and field potentials in the hand motor representation areas, were able to learn to control a computer cursor in several directions [2], as well as a robotic arm performing three-dimensional reach and grasp movements $[1,3]$. These procedures are impressive and extremely significant for their

Electronic supplementary material The online version of this article (https://doi.org/10.1007/s13311-019-00816-2) contains supplementary material, which is available to authorized users.

Andrea Caria

andrea.caria@unitn.it

1 Department of Psychology and Cognitive Sciences, University of Trento, Corso Bettini 33, 38068 Rovereto, Italy

2 Istituto di Ricovero e Cura a Carattere Scientifico, Fondazione Ospedale San Camillo, Venice, Italy

3 Institut für Medizinische Psychologie und Verhaltensneurobiologie, Universität Tübingen, Tübingen, Germany
4 Brain and Language Laboratory, Department of Clinical Neuroscience, University of Geneva, Geneva, Switzerland

5 Institute of Biological and Medical Engineering, Pontificia Universidad Católica de Chile, Santiago, Chile

6 Department of Psychiatry, Section of Neuroscience, School of Medicine, Pontificia Universidad Católica de Chile, Santiago, Chile

7 Laboratory for Brain-Machine Interfaces and Neuromodulation, School of Medicine, Pontificia Universidad Católica de Chile, Santiago, Chile

8 Health Technologies Department, TECNALIA, San Sebastian, Spain 
neuroscientific and clinical implications but are not shown to directly promote restoration or improvement of hand/arm motor functions in paralyzed patients.

Alternatively, other BMI systems allow to bypass motor system disruption and to stimulate functional recovery and neuroplasticity by coupling contingent neuroelectric signals related to motor commands with movements of assistive prosthetic devices that crucially provide afferent and proprioceptive feedback to the somatosensory cortex. Increasing evidence shows that chronic stroke patients with minimal residual hand movements can control prosthetic devices fixed to the paretic upper limb through BMI-mediated learned regulation of motor-related oscillatory brain activity [4-10]. This approach is particularly important for severe stroke patients with minimal or no residual motor functions, which are usually excluded from intensive therapeutic intervention and rehabilitation such as constrained induced movement therapy, bilateral arm training, or robot-assisted training, as they rely on the existence of residual motor functionality [4, 11-15]. In these patients, contingencies between overt actions and consequences can no longer be used to drive reorganization within functional brain networks, making them prone to devolution towards a maladaptive state indicative of learned disuse $[16,17]$. Severely impaired chronic stroke patients often show increased activity in the unaffected hemisphere during upper limb movement, which in most cases represents vicariation of motor function $[18,19]$. However, the disuse of the ipsilesional hemisphere and compensatory overuse of the healthy contralesional hemisphere have been also associated with abnormal interhemispheric inhibition from the intact to the damaged hemisphere [20-22], which is even more pronounced in the case of severe motor impairment [23]. Typically, functionally significant motor recovery is associated with a rebalanced sensorimotor cortex activity towards the ipsilesional/contralateral hemisphere [24-29].

Stimulation and reinforcement of the remaining preserved structures in the affected hemisphere might thus represent a more neurobiologically appropriate approach to foster recovery. A previous double-blind randomized placebo-controlled study demonstrated the clinical efficacy of EEG-BMI for stroke rehabilitation [15]. Pilot studies also suggested that EEG-BMI-based intervention exploiting ipsilesional motor commands might indeed stimulate adaptive functional and structural reorganization in patients after stroke $[12,13,30]$. More recently, changes in functional and structural brain reorganization in stroke patients showing upper limb motor recovery were demonstrated after EEG-brain-computer interface (BCI) [8-10, 31, 32]. The capability of non-invasive neuromodulation techniques of inducing brain plasticity has been further indicated by other instrumental learning approaches revealing for instance structural and functional connectivity reorganization after short motor imagery-based neurofeedback training [33]. Here, structural and functional motor network connectivity was assessed in partially recovered chronic stroke patients after EEG-BMI training. We hypothesized that reinforcement of the ipsilesional sensorimotor activity contingent on upper limb movement attempts induced rebalancing of interhemispheric network interactions towards the re-establishment of original motor system neurophysiology.

\section{Methods}

\section{Participants}

Patients in both experimental and sham control groups were recruited via public information (German stroke associations, rehabilitation centers, hospitals) all over Germany. All participating patients fulfilled the following criteria: paralysis of 1 hand with no active finger extension after unilateral stroke; interval since stroke of at least 10 months; age between 18 and 80 years; no psychiatric or neurological condition other than stroke; no cerebellar lesion or bilateral motor deficit; no pregnancy; no claustrophobia; no epilepsy or medication for epilepsy treatment during the last 6 months; eligible to undergo magnetic resonance imaging (MRI); ability to understand and follow instructions. Exclusion criteria included depression or other psychological impairment, active finger extension, more than 1 stroke, no motivation to participate after detailed information of the study (mostly due to geographic distance), and other health complications such as brain trauma or cancer. Patients were pseudo-randomly assigned to experimental $(n=$ $16)$ and control $(n=14)$ groups; group assignment was blinded for all participants and for the scientific clinical personnel so that none of the patients or therapists was able to characterize group assignment (double-blind). Groups were matched for age, gender, lesion location, and functional capacity scores at time of inclusion (see supplementary material). One patient was excluded from the study for unreliability of clinical outcome measurements. The study was conducted at the University of Tübingen in Germany. Informed consent, including informed consent to publish identifying information/images in an online open-access publication, was obtained from all patients involved. The study was approved by the ethics committee of the Faculty of Medicine of the University of Tübingen. The methods carried out in this work are in accordance with the approved guidelines and regulations.

\section{BMI Intervention and Behavioral Outcome Measures}

Thirty participants underwent a 4-week EEG-BMI-based intervention for upper limb rehabilitation. Movements of the arm/hand were directly dependent upon sensorimotor oscillations of 8 to $13 \mathrm{~Hz}$ recorded over the ipsilesional sensorimotor 
cortex. Patients in the experimental ( $n=16$, contingent feedback) and control ( $n=14$, sham feedback) groups were instructed that during training, they had to try to reach, grasp, and bring to their lap an imaginary apple (thus involving finger extension during the reach and grasp movement [34]) assisted by 2 separate hand and arm orthoses (Fig. 1). During the first training sessions (no. of sessions, $6.5 \pm 3.8$, experimental group; $5.1 \pm 3.9$, control group), a robotic device (Motorika, Israel) connected to the active EEG electrodes over the motor ipsilesional cortex supported upper arm reaching movements of the paretic arm. The patient's arm, strapped to the robot's arm, moved forward for $5 \mathrm{~s}$ if the patient produced an event-related desynchronization (ERD) of his/her preidentified sensorimotor rhythm (SMR). An average of 16 runs of 11 trials, each separated by few seconds of rest and several breaks of few minutes, were performed for each session (day of training). The robot training was dependent on the patient's ability to move the upper arm and reach towards the front. If the patient was able to perform such movement, the training started directly using the hand robotic orthosis. During the consecutive hand-orthosis training sessions (no. of sessions, $11 \pm 3.8$ experimental group; $13.1 \pm 3.9$ control group), the paretic hand was inserted in the slings of an orthosis and patients were instructed to try to open their hand after an auditory trigger signal for $5 \mathrm{~s}$ even if the fingers did not actually follow their intention. Similar to previous arm sessions, ERD of the ipsilesional SMR under a certain threshold opened the hand in the orthotic device for $5 \mathrm{~s}$. In the experimental group, SMR activity was linked to active reaching and grasping movements and finger extension and flexion (or attempt to move) (contingent feedback), whereas in the control group, random movements not linked to brain rhythms controlled the orthoses (see Ramos-Murguialday et al. [15] for an exhaustive description of the EEG-BMI training and results). A demographic summary of the experimental and control groups can be found in Table 1. A comprehensive battery of motor function assessment instruments, including the Fugl-Meyer Assessment (FMA) Scale, the Ashworth Scale, the Motor Activity Log (MAL) [35], and a Goal Attainment Scale (GAS) [36], was given twice, before ( 8 weeks (Pre1) and 1 day (Pre2) before BMI training), and once immediately after treatment (Post). The modified upper limb combined arm and hand motor scores from the Fugl-Meyer Assessment Scale was used as the primary behavioral outcome measure [15]. The combined FMA (cFMA) score is obtained by adding FMA hand/finger score (24 points) and FMA arm score (30 points) [37].

\section{MRI Data Acquisition}

The MRI recordings were performed with a 3-T Magnetom (Tim Trio, Siemens, Erlangen) whole-body scanner using a standard 12-channel head coil. Each patient participated in multiple MRI measurements: 8 weeks before training (Pre1), 1 day before training (Pre2), and few days after training (Post). A T1-weighted anatomical MR image was acquired using a 1$\mathrm{mm}$ isotropic MPRAGE sequence with the following parameters: $\mathrm{TR}=2300 \mathrm{~ms}, \mathrm{TE}=3.03 \mathrm{~ms}, \mathrm{TI}=1100 \mathrm{~ms}$, flip angle $=$ $8^{\circ}, \mathrm{FOV}=256 \times 256$, matrix size $=256 \times 256$, number of slices $=176$, slice thickness $=1 \mathrm{~mm}$, bandwidth $=130 \mathrm{~Hz} /$

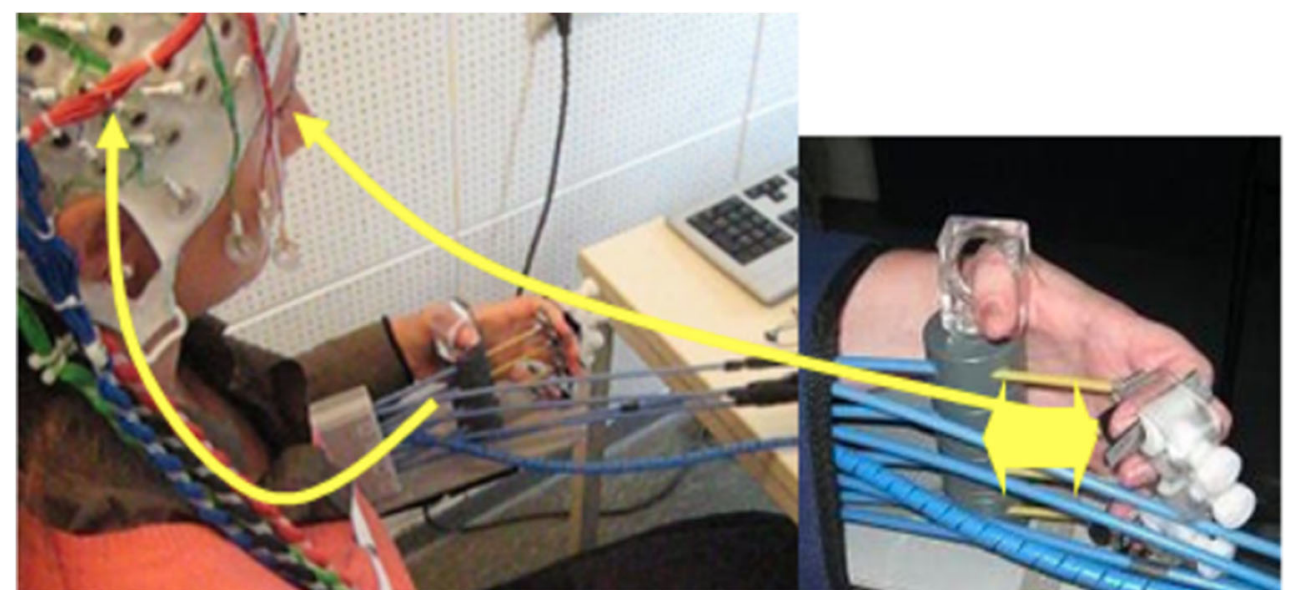

Fig. 1 BMI setup. Patients were required to learn SMR desynchronization, measured with a 16-channel EEG system overlying the ipsilesional motor cortex. The EEG system was coupled with either hand or arm orthosis that supported reaching and grasping movements during BMI training. BMI training aimed to improve upper arm extension and flexion when using arm orthosis (reaching) and finger extension and flexion when using hand orthosis (grasping). The sensorimotor rhythm (SMR) power recorded from the ipsilesional electrodes was translated into movement of the orthosis. A threshold calculated as the point of equal distance to the mean of the power distribution during rest and motor intention calculated over the last $15 \mathrm{~s}$ defined rest and motor intention classification areas. When the SMR power was continuously in the motor intention classification area for $200 \mathrm{~ms}$, the orthosis moved; the orthosis stopped when returned to the rest classification area for $200 \mathrm{~ms}$ and maintained the previous state otherwise. The same BMI principle was applied when training reaching movements with the arm orthosis. The experimental group received contingent feedback, linking SMR activity with movement execution supported by hand and arm orthoses; the control group received sham feedback, in which random movement of hand and arm orthoses was not linked to brain rhythms 
Table 1 Means and standard deviations of demographic data and functional scores of the 2 patients groups at the time of enrolment in the study. In the experimental group, brain activity moved the orthoses; the control group received random orthosis movements not linked to control of oscillatory brain activity. Lesion side indicates damaged hemisphere. Motor part of the modified upper limb cFMA (hand and arm parts combined having a maximum score of 54 points) and Goal Attainment Scale (GAS) scores are presented for both groups. Training duration indicates the number of runs during BMI training. One run contains 11 trials of $5 \mathrm{~s}$ in which the patients were able to move the orthosis using the brain-machine interface system. None of the differences of baseline measures between the experimental and control groups was significant

\begin{tabular}{lllllrr}
\hline Group & Age (years) & Months since stroke & Lesion side & cFMA & GAS & Training duration \\
\hline Experimental & $49.3 \pm 12.5$ & $69 \pm 43$ & $8 \mathrm{R} / 8 \mathrm{~L}$ & $11.16 \pm 1.73$ & $0.88 \pm 0.67$ & $275 \pm 25$ (runs) \\
Control & $50.3 \pm 12.2$ & $64 \pm 68$ & $8 \mathrm{~L} / 6 \mathrm{R}$ & $13.29 \pm 2.86$ & $0.63 \pm 0.51$ & $291 \pm 17$ (runs) \\
\hline
\end{tabular}

Px. Diffusion tensor imaging (DTI) data were acquired using a single echo planar imaging (EPI) sequence; 30 diffusionweighted images $\left(b=800 \mathrm{~s} / \mathrm{mm}^{2}\right)$ and a reference T2weighted image with no diffusion weighting $(b=0 \mathrm{~s} /$ $\mathrm{mm}^{2}$ ) were obtained with the following acquisition parameters: voxel size $=1.86 \times 1.86 \times 2.5 \mathrm{~mm}$, gap $=$ $2.5 \mathrm{~mm}, \mathrm{TR}=7900 \mathrm{msec}, \mathrm{TE}=89 \mathrm{~ms}, \mathrm{FOV}=240 \times 240$ $\mathrm{mm}$, flip angle $=90^{\circ}$, matrix size $=128 \times 128$, slices $=60$ transversal. The acquisition scheme was repeated 3 times to improve the signal-to-noise ratio. Functional MR images, aligned in axial orientation, were acquired using EPI technique: $\mathrm{TR}=2000 \mathrm{~ms}, \mathrm{TE}=30 \mathrm{~ms}$, flip angle $=90^{\circ}$, $\mathrm{FOV}=210 \mathrm{~mm}$, matrix size $=64 \times 64$, interslice gap $=$ $0.75 \mathrm{~mm}$, slices $=28$, slice thickness $=3 \mathrm{~mm}$. Each fMRI session consisted of 4 runs (190 volumes $\times$ run) of visually and auditory-cued attempted/executed (12 s) and imagined (12 s) flexion-extension of the fingers, with either the affected or the unaffected hand, alternated with rest (12 s). Healthy and paretic hand movements were monitored during each condition by simultaneous EMG measurement via 3 pairs (bipolar) MR compatible surface EMG electrodes placed over the biceps and the extensor digitorum and extensor radialis muscles. In order to minimize head movements, 2 foam cushions were positioned around participant's head.

\section{DTI Data Analysis}

In our study, changes in structural connectivity after BMI training were explored by assessing longitudinal variations of fractional anisotropy (FA) and mean diffusivity (MD) [38]. FA reflecting the diffusion signal directional dependence of the brain tissue structure in relation to the degree of anisotropy (represented as an ellipsoid) ranges from 0 (isotropic) to 1 (anisotropic) for increasingly highly oriented fibers. Mean diffusivity describes the magnitude of water diffusion and is the average of the eigenvalues representing the amount of diffusivity along the 3 main orthogonal directions $([\lambda 1+$ $\lambda 2+\lambda 3] / 3)$. FA is sensitive to changes of several white matter properties such as axonal myelination, diameter, density, and orientation of fibers [39, 40], and it has been extensively used to describe brain remodeling after stroke and during functional recovery [41-45], also mediated by BCI training [10, 31]. However, both MD and FA do not completely describe full tensor distribution being rotationally invariant scalar indices that can be equally associated with different eigenvalue combinations. Thus, differences of diffusion metrics such as axial (parallel) diffusivity $(\mathrm{AD}=\lambda 1)$ and radial (perpendicular) diffusivity $(\mathrm{RD}=[\lambda 2+\lambda 3] / 2)[38]$ were also assessed comparing mean values in white matter regions using bootstrap analysis (5000 bootstrap samples, 95\% bias-corrected and accelerated confidence interval) as implemented in SPSS (Release 24.0.0.0). Previous studies showed changes of RD in relation to fiber myelination, whereas AD appears to be sensitive to axonal degeneration associated with changes in axonal density or caliber [42]. FA increases have been typically observed as a result of augmented axial diffusivity and/or diminished radial diffusivity [43, 44].

\section{DTI Data Preprocessing}

Diffusion data were analyzed using the DTI \& Fiber Tools toolbox (Freiburg, Germany) (https://www.uniklinikfreiburg.de/mr-en/research-groups/diffperf/fibertools.html).

Skull striping was first applied to delete non-brain tissue from the image. Eddy currents and head motion correction were performed using affine registration to the b0 image. The diffusion gradient directions were adjusted. A voxelwise calculation of the tensor matrix and FA and MD maps were then obtained for each subject. All resulting images were visually inspected to verify proper pre-processing results. Before normalization, stroke lesions were masked by using individual lesion masks created manually on axial slices of a T1-weighted images using the MRIcron software package (see supplementary figure 2). Normalization to standard space was accomplished using the SPM8 normalization function. Co-registration of the International Consortium for Brain Mapping (ICBM)-template [45] image with the realigned FA and MD images separately using normalized cross-correlation was followed by application of the deformations estimated for the normalization of that co-registered ICBM-DTI template back to standard space to the realigned FA and MD images. 
A three-dimensional Gaussian smoothing kernel $(2 \mathrm{~mm}$ FWHM) was applied to the resulting images. Images were left-right flipped when necessary using the reorient function of SPM8 so that the right side of the image always corresponded to the ipsilesional hemisphere.

\section{DTI Data Classification}

The aim of DTI data analysis was to evaluate a classifier's performance in discriminating experimental group from sham control group based on changes of DTI metrics (FA and MD) after BMI training. Analysis of DTI data was based on a support vector machine [46] (linear SVM classifier available on MATLAB Statistics Toolbox) classification of DTI measures, as this approach, that considers the inherent multivariate relationships and spatial dependency of white matter fiber bundles [47], proved to increase sensitivity in detecting morphological alterations associated with several neurological conditions including stroke [48-52]. For instance, changes of DTI measures in the corticospinal tract (CST) and periventricular white matter associated with stroke outcome were detected with multivariate analysis but not with univariate methods [48]. SVM-based classification included the following steps: feature selection, classifier training and testing, and evaluation of classification performance.

\section{Feature Selection}

Feature selection is a technique that by reducing data dimensionality through the identification of a subset of relevant features and the exclusion of redundant or irrelevant features permits to reduce overfitting and to increase signal-to-noise ratio. Feature selection was performed through a leave-oneout cross-validation approach repeated $n$ times, in which $n$ is the number of patients in the experimental and control groups. Feature selection was performed for each cross-validationfold by extracting the Fisher score $S_{\mathrm{i}}$ of each voxel, calculated as $\sum n_{\mathrm{j}}\left(\mu_{\mathrm{ij}}-\mu_{\mathrm{i}}\right)^{2} / \sum n_{\mathrm{j}} * \rho_{\mathrm{ij}}^{2}$ in which $\mu_{\mathrm{ij}}$ and $\rho_{\mathrm{ij}}$ represent the mean and the variance of the $i$ th feature in the $j$ th class, respectively, $n_{\mathrm{j}}$ is the number of instances in the $j$ th class, and $\mu_{\mathrm{i}}$ is the mean of the $i$ th feature, and applying them to a searchlight approach [53] using cubes with a side-length equal to 3 voxels $[54,55]$. Feature selection based on the Fisher score aims at potentiating classification on the basis of high interclass deviations and low intraclass variations. A good feature has in fact large separation between classes and high uniformity within each class. This process resulted in an output classification label for each patient, which could be either positive $(+1$, sham control group) or negative $(-1$, experimental group). The output was then compared to the input label, and in the case of correct matching the classification was considered successful. This process identified voxels (out of 983,040 voxels) that increased classification accuracy between the experimental and control groups.

\section{Classifier Training and Testing and Performance Evaluation}

Robustness of the classifier was assessed by separating training and testing data sets using leave-one-out cross-validation approach. Training and data classification were performed using a SVM classifier with linear kernel available on MATLAB Statistics Toolbox. The linear SVM is represented by the weighted sum of the input features plus a bias term:

$f(x)=w T x+\mathrm{b}$,

in which $x$ is the input feature's vector, $w$ is the weight vector, and $b$ is the bias value. C parameters estimation were $n /(2 * n 1)$ for the data points of the experimental group and $n /(2 * n 2)$ for the data points of the control group, in which $n 1$ is the number of elements of the experimental group, $n 2$ is the number of elements of the control group, and $n=n 1+n 2$. Positive weights indicated higher values for the sham control group, whereas negative weights indicated higher values for the experimental group.

Classification of Pre2-Pre1 difference images and PostPre2 difference images with all possible label permutations was also performed to ensure results against spurious outcomes. The final analysis of Post-Pre2 difference images was executed with 30 samples (experimental group $=16$, sham group $=14$ ). Classification of images capturing withinsubject FA or MD changes into treatment groups constitutes a data-driven method to identify differences between the effects of treatment-group membership based on differences of white matter metrics using robust machine learning methods. Evaluation of classifier's performance was then based on confusion matrix indicators including accuracy, sensitivity, and specificity. Classification accuracy corresponded to the percentage of correct experimental group classification, sensitivity to the percentage of correct experimental group classification, and specificity to the percentage of correct sham control group classification. A binomial test was used to assess significance of classification accuracy.

\section{fMRI Data Preprocessing and Analysis}

fMRI data analysis was performed using SPM12. EPI volumes of the pre- and post-intervention fMRI sessions were realigned, slice time corrected, and anatomically co-registered. Before spatial normalization (MNI template) and smoothing (6-mm FWHM Gaussian kernel), stroke lesions were masked using individual ROIs [56] (see supplementary figure 1). Images of right-hemispheric stroke patients were flipped to the left hemisphere so that all right images corresponded to the ipsilesional hemisphere. Patients with lesions in primary sensorimotor regions were excluded from group analysis. Hemodynamic response amplitudes were 
estimated using standard regressors, constructed by convolving a boxcar function, for each of the 3 different conditions (executed/attempted movement, imagined movement, and rest), with a canonical hemodynamic response function using standard SPM12 parameters. The time series in each voxel were high-pass filtered at $1 / 128 \mathrm{~s}$ to remove low-frequency drifts. First-level contrast images of executed and imagined movement conditions compared with those of rest were created for each session and group. Movement parameters were also included into the general linear model (GLM) as covariates to account for head motion artifacts. These images were then entered into a second-level random effects analysis and post hoc $t$ test analysis to allow inferences across participants that generalize to the population. A flexible factorial design with 2 factors, Time (Pre1, Pre2, and Post) and Group (experimental and control), considering movement execution condition was adopted. In our random effects model, we allowed for violations of sphericity by modeling non-independence across images from the same subject and unequal variances between conditions and subjects as implemented in SPM12. The resulting statistical maps were thresholded at $p<0.005$ ( $t>$ 2.92) [57-59] corrected for multiple comparison using probabilistic threshold-free cluster enhancement $[60,61]$, an approach that integrates cluster information into voxel-wise statistical inference to enhance detectability of neuroimaging signal. All brain coordinates are reported in MNI atlas space. A lateralization index (LI) expressed as the normalized difference between the number of active voxels in the ipsilesional and contralesional hemispheres [62] was calculated for the paretic and healthy hand before and after BMI training sessions. LI was calculated by selecting an inclusive mask of primary motor cortex (BA4), premotor cortex (including SMA, BA6), and primary somatosensory cortex (BA3), masking out the midline $( \pm 5 \mathrm{~mm})$, and by using a combination of clustering (smoothing $=2 \times$ voxel size) and variance weighting approaches to determine reliability of activations and remove outliers [62]. LI yields a value of 1 or -1 when the activity was purely contralesional or ipsilesional respectively. The differences of LI calculated individually were correlated with FA changes.

\section{Functional Connectivity Analysis}

Motor network functional connectivity was assessed in order to elucidate and possibly support the convergent BMI trainingrelated differences in FA and BOLD activity. Connectivity analysis was performed using the CONN functional connectivity toolbox (Release 18.a, http://www.nitrc.org/projects/conn) [63]. Functional connectivity was estimated using a weighted GLM for weighted temporal correlation measures (weighted seedbased connectivity) between BOLD time series in a defined seed area and whole brain voxels during paretic limb movement execution. This data-driven approach permits exploration of functional relationships among all nodes of brain motor network, being not dependent of priori assumptions and arbitrary selection of limited number of regions that are instead necessary in model-based effective connectivity methods such as dynamic causal modeling.

Before computing connectivity analysis, denoising was applied to reduce motion and physiological and additional artifactual effect from the BOLD signal. Noise reduction was performed based on the available CompCor method that includes the principal components ( 5 each) of WM and CSF time series as nuisance covariates; WM and CSF were identified via segmentation of the anatomical images with SPM12. Subjectspecific lesion masks were included as additional covariates to regress out. The 6 head motion parameters derived from spatial motion correction were also added as confounds. Band-pass filtering with a frequency window of 0.008 to $0.09 \mathrm{~Hz}$ was applied. Seed-to-voxel maps were then created for each participant. Two different seeds, represented by two 9-mm spherical regions in the parietal cortex $(x, y, z=-23,-$ $56,51)$ and supplementary motor area $(x, y, z=-13,10,63)$ in the contralesional hemisphere, were considered. These regions, masked with subject-specific estimated gray matter mask, were defined based on the observed between-group training effect activation maps during paretic hand movement execution (see the "Results" section). In addition, functional connectivity of the contralesional primary motor area (M1, $x, y, z=-30,-16$, 54, 9-mm radius - coordinates corresponding to the main BOLD cluster during healthy hand movement) was also explored as previous studies showed inhibitory influences from contralesional to ipsilesional M1 during paretic hand movements [22, 64-66]. Separate bivariate-correlation analyses were used to determine the linear association of the BOLD time series between each of the 3 "seed" regions separately and whole brain areas on each participant. Individual seed-tovoxel maps were then entered into a second-level analysis. Between-group temporal correlations were analyzed considering paretic hand movement condition during Post $v s$ Pre2 and Pre $2 v$ Pre1. The resulting connectivity maps were thresholded at $p<0.005(t>2.92)$, corrected for multiple comparison using probabilistic threshold-free cluster enhancement [60,61]. Assessment of resulting changes in positive or negative correlations was performed by creating a mask of the resulting significant clusters of interest of the interaction effect and then by exploring connectivity values for each cluster of each session.

\section{Results}

\section{Clinical Outcome}

No significant differences were measured for upper limb motor functions between experimental and control groups at baseline. A 2-way mixed model ANOVA (with independent 
measures on group and repeated measures on time) showed a significant Time (Pre2 and Post) $\times \operatorname{Group}\left(F_{1,28}=6.294, p=\right.$ $0.018)$ interaction and a significant effect of time $\left(F_{1,28}=\right.$ 9.588, $p=0.004)$ on cFMA scores. There was no main effect of group $\left(F_{1,28}=0.034, p=0.855\right)$. A significant improvement of the cFMA was observed in the experimental group $\left(\right.$ mean \pm SE Pre2 $=11.16 \pm 1.73$, Post $=14.56 \pm 1.95, t_{1,15}=$ $6.049, p<0.001$ ) but not in the sham control group (mean \pm $\mathrm{SE}$, Pre2 $=13.29 \pm 2.86$, Post $=13.64 \pm 2.91, t_{1,13}=0.316$, $p=0.757)$. None of the other behavioral measures showed any differences between groups (see Ramos-Murguialday et al. [15] for a complete description of clinical outcome). In addition, motor function outcomes 6 months after the intervention (Post2) indicated long-lasting improvements [67]. In fact, in the experimental group, only cFMA scores were significantly higher in Post2 (13.44 \pm 1.96$)$ as compared with Pre2 $(p=0.015)$ session and no significant difference was measured between Post and Post 2 sessions.

\section{DTI Data Classification}

DTI data analysis showed successful classification of the experimental and control groups based on individual FA images representing Pre and Post-training difference (2-sided binomial test, $p=0.0002$ ), with an average accuracy of $82.8 \%$ (sensitivity, $75 \%$ positive effect of treatment, i.e., experimental group correctly identified; specificity, $92.3 \%$ negative effect of treatment, i.e. control group correctly identified). Sixteen label permutations were performed to compute a chance level of classification and ensure that the classifier would not classify any arbitrary distinction into classes with equal accuracy merely due to the high number of available features and low number of patients. The computed chance level resulting in an average accuracy of $48.9 \%$ ( \pm SD $8.8 \%$; average sensitivity, $57.8 \%, \pm 22.4 \%$; average specificity, $37.9 \%, \pm 20.7 \%$ ) indicated that the successful classification of Post-Pre2 FA change images into the experimental group does not occur due to an imbalance of available features and random variations.

Classification accuracy of the experimental and control groups based on individual MD images representing Pre and Post-training did not reach significance level indicating no specific treatment effect on white matter mean diffusivity (2sided binomial test, $p>0.05)$. All FA and MD classification results are displayed in Fig. 2.

FA-related spatial analysis of the selected voxels discriminative for the treatment effect revealed that voxels were located in subcortical white matter tracts within posterior and mainly contralesional brain regions. Specifically, the experimental group showed FA decrease whereas the control group showed FA increase in the same sites (see supplementary figure 1). The most significant decreases of FA were observed in the splenium (SPL) and body of corpus callosum (BCC) (subregions corresponding to transcallosal fibers interconnecting parietal cortex - SPL - and dorsal premotor cortex, pre- and supplementary motor areas-BCC [68, 69]), and in the contralesional hemisphere in the posterior limb of the internal capsule (PLIC), posterior thalamic radiation (PTR), and superior corona radiata (SCR) (Fig. 3). Classification analysis of individual Pre2-Pre1 FA and MD images into the experimental and control groups did not show significant effects with an average accuracy of $65.2 \%$ (FA) and $56 \%$ (MD), indicating that identification of treatment group membership was performed close to chance level.

Additional univariate analysis of DTI metrics showed no significant training-related differences ( $p>0.05$, Fig. 4 top) of $\mathrm{FA}, \mathrm{MD}, \mathrm{AD}$, and $\mathrm{RD}$ within each of the white matter regions differentiating experimental and control groups. A significant FA decrease $(p=0.039)$ and a tendency of RD increase ( $p=$ $0.054)$ was observed considering all the abovementioned white matter regions together (Fig. 4 bottom).

\section{FA Relationship to Clinical Outcome}

A negative correlation was measured between increased motor functions as evidenced by increased cFMA and FA decrease in the splenium (Pearson's $r=-0.775$, df $=$ 13, $p<0.01,2$-tailed) and in the contralesional posterior thalamic radiation (Pearson's $r=-0.718, \mathrm{df}=13, p<0.01$, 2-tailed) in the experimental group. No significant correlation between cFMA and FA changes was measured in the control group.

\section{fMRI Data}

We observed a significant interaction effect between Group and Time on brain activation for paretic hand movement execution as compared with rest, thereby showing a differential training effect between experimental and control groups. This interaction was investigated using separate t-contrasts. The experimental group as compared with the control group showed decreased BOLD activity in the contralesional superior parietal cortex (SPC: $x, y, z=-23,-56,51 ; k=52$ ) and the supplementary motor area (SMA: $x, y, z=-13,10,63 ; k=11)$ during paretic hand motor execution after training (Post $>$ Pre2, Fig. 5). The experimental group showed predominant unilateral brain activity when the task was performed with the healthy hand in Pre1, Pre2, and Post, with maximum peaks in the sensorimotor regions contralateral to the movement (Pre1: MNI $x, y, z=-42,-33,61$; Pre2: $x, y, z=-30,-16,54$; Post: $x, y, z=-39,-26,57)$; no significant differences were measured over sessions. Bilateral brain activity was observed during the affected hand task with maximum peaks in the contralateral/ipsilesional primary sensorimotor areas before and after BMI training (Pre1: $x, y, z=25,-16,73$; Pre2, MNI $x, y, z=30,-23,72$; Post: $x, y, z=26,-23,69)$. No movements were observed and recorded with 
FA
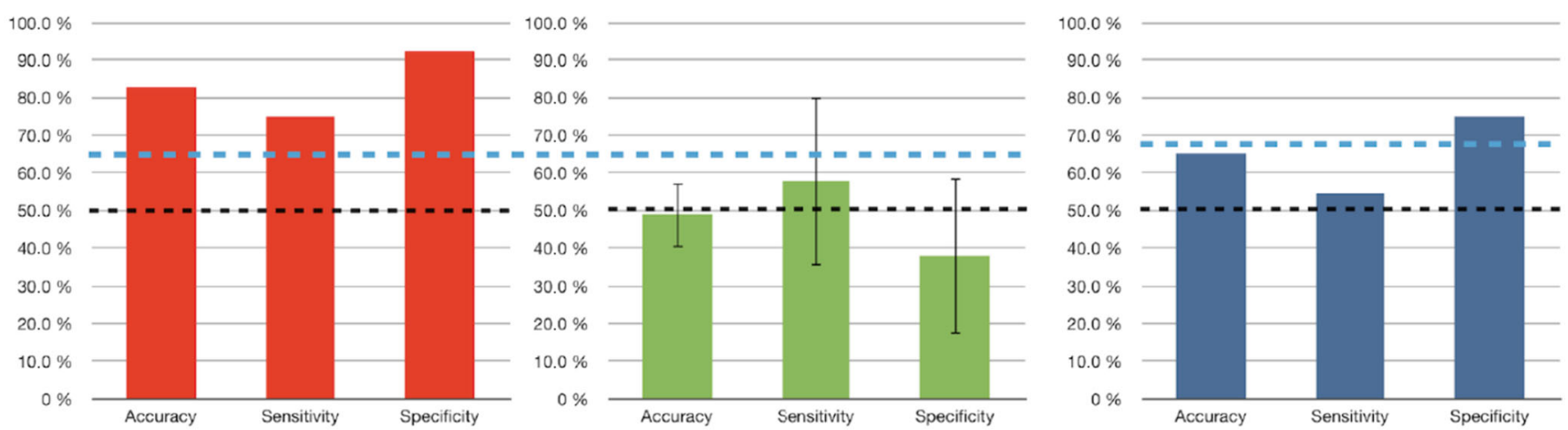

MD
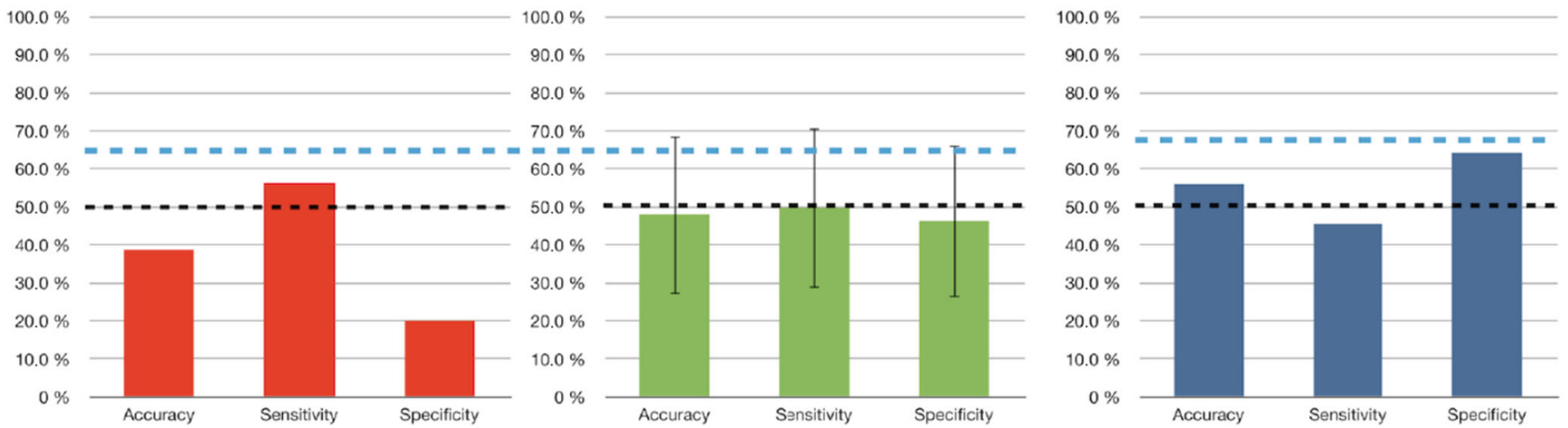

Fig. 2 FA and MD changes in classification performance. Left, PostPre2 classification (red). Center, label permutations of the treatment effect classification (green). Test statistic was compared with the distribution of permutation values, which are computed similarly to the test statistic but under a random rearrangement of the labels of the data

set. Right, Pre2-Pre1 classification (blue). The dotted black line indicates the $50 \%$ chance level; the dotted blue line indicates the level in which classification accuracy is considered statistically significant based on binomial test. $Y$-axis, percentage of correct classification

electromyography (EMG) during motor imagery and rest condition and no mirror movements or involuntary co-contraction was observed or measured during the actual movement condition in both the experimental and control groups.
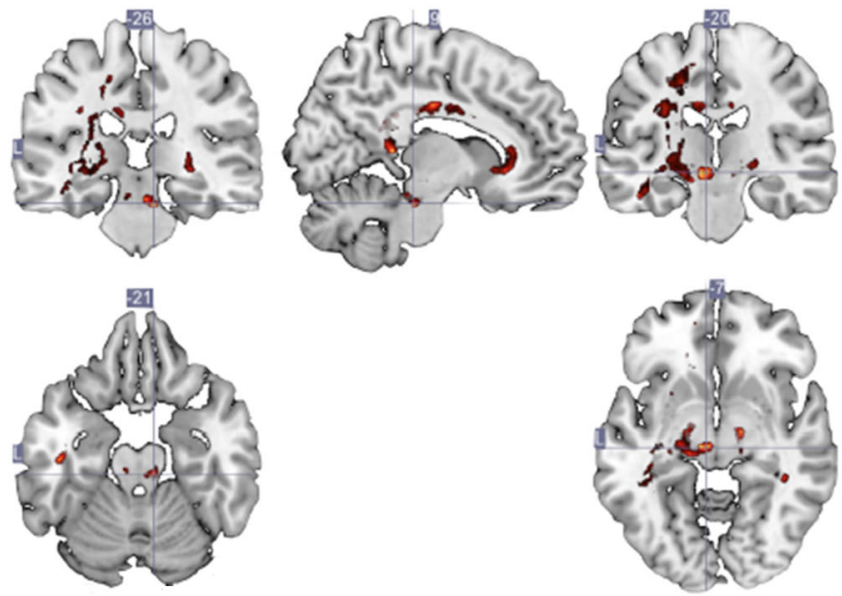

Fig. 3 SVM weight vector for each voxel selected as feature. A linear SVM is the weighted sum of the input features plus a bias term: $f(x)=$ $w T x+b$, in which $x$ is the input feature's vector, $w$ is the weight vector, and $b$ is the bias value. Heatmap color-coding indicates voxel-weight; maximal weights were located in posterior and middle midline corpus callosum structures (right) as well as parts of the geniculate fibers and
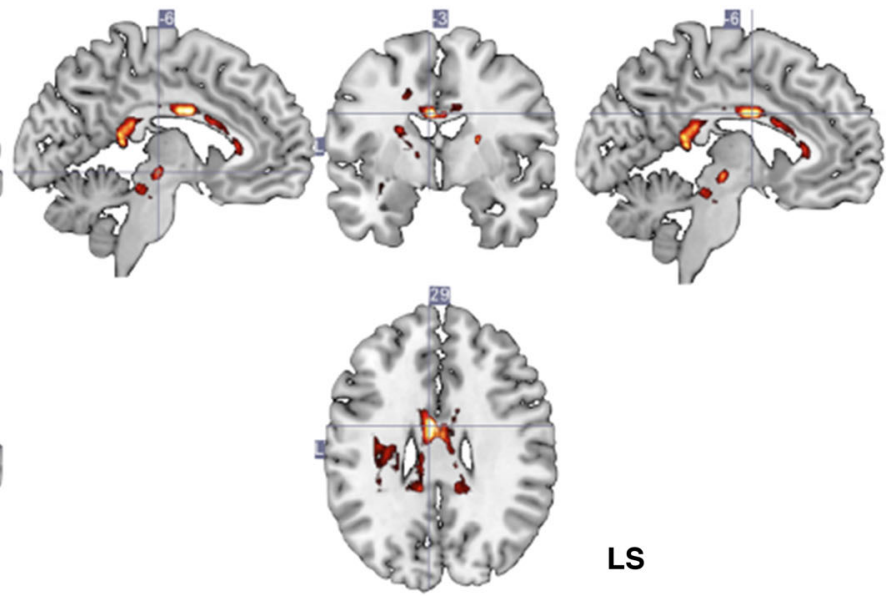

LS

internal capsule (left and middle). Analysis of the within-group white matter changes reveals that patients in the experimental group showed a decrease of FA in the splenium and body of the corpus callosum, posterior limb of the internal capsule (contralesional), posterior thalamic radiation (contralesional), and superior corona radiata. $\mathrm{LS}=$ lesional side 
Fig. 4 Top, Pre- and post-training values of $\mathrm{FA}, \mathrm{MD}, \mathrm{AD}$, and $\mathrm{RD}$ (mean $\pm \mathrm{SD}$ ) in the splenium and body of the corpus callosum and in the contralesional posterior limb of the internal capsule, posterior thalamic radiation, and superior corona radiata. Bottom, $\mathrm{FA}, \mathrm{MD}, \mathrm{AD}$, and $\mathrm{RD}$ values $($ mean $\pm \mathrm{SD})$ averaged over all the regions
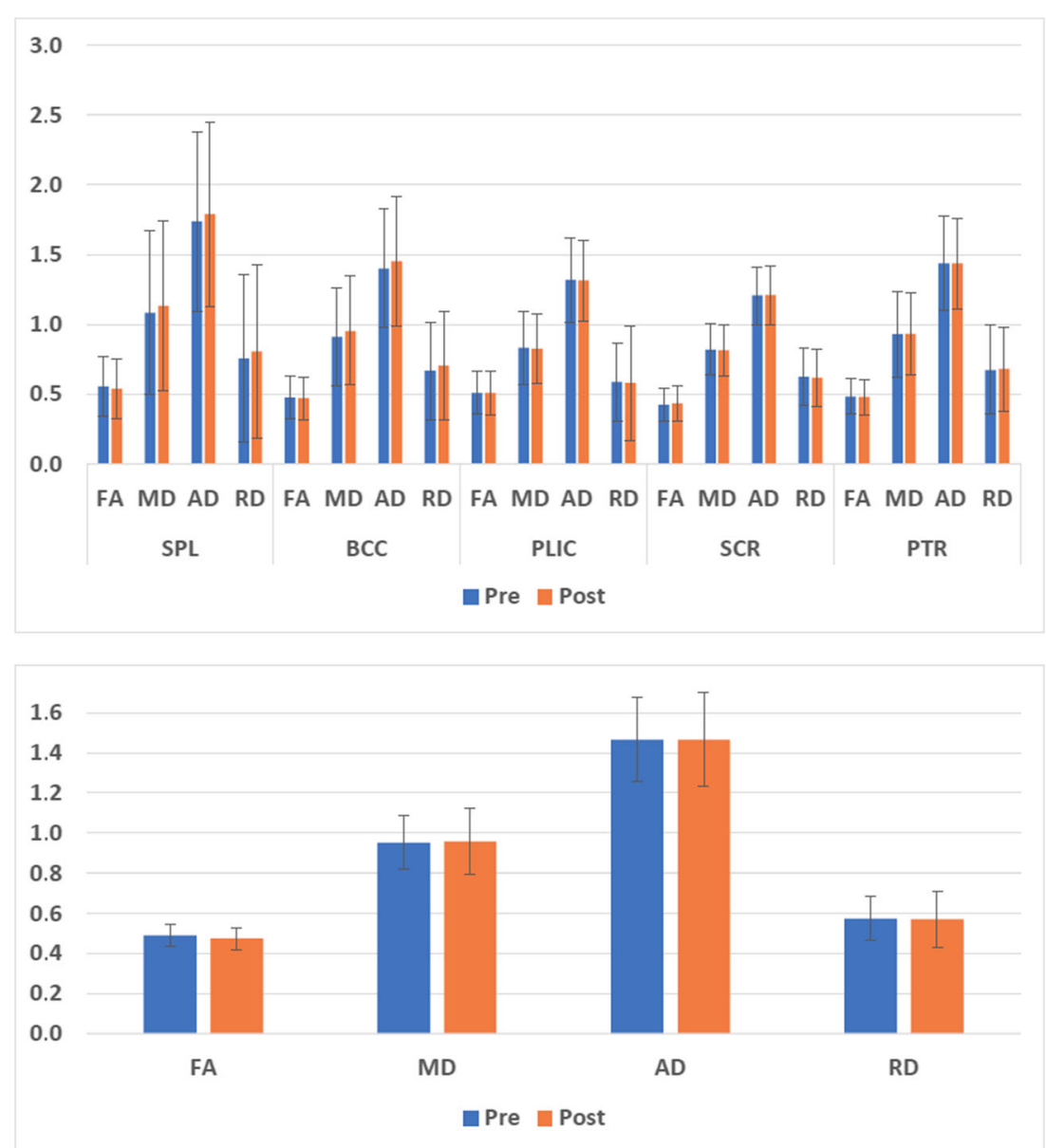

\section{Functional Connectivity}

In the experimental group with respect to the control group, the contralesional SMA showed decreased positive coupling (Fig. 6 top, warm colors) with contralesional cerebellum ($26,-50,8)$, bilateral lingual gyrus $(-26,-10,70 ; 18,-46,-$ $4)$, and contralesional premotor cortex $(-30,-14,-70)$ and decreased negative coupling (Fig. 6 top, cool colors) with the ipsilesional premotor/motor cortex $(58,0,26)$, the putamen $(28,-4,12)$, and the ventromedial prefrontal cortex $(6,22$, -16) during paretic hand movement, after BMI training (Pre2 > Post) (Fig. 6, cool colors).
The contralesional M1 of the experimental group as compared with the control group showed decreased negative coupling (Fig. 6 bottom, cool colors) with ipsilesional premotor cortex $(42,2,44)$, primary motor cortex $(46,-14,54)$, cingulate cortex $(2,30,30)$, and parietal cortex $(40,-36,48)$ and decreased positive coupling (Fig. 6 bottom, warm colors) with contralesional $(-6,-30,-12 ;-40,-54,-6)$ and ipsilesional $(22,-74,-34 ; 44,-60,34)$ cerebellum, after BMI training. Connectivity value exploration in the experimental group over sessions revealed that for both contralesional SMA and M1, decreased positive coupling corresponded to changes of correlation from positive (Pre2)
54

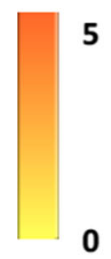

58

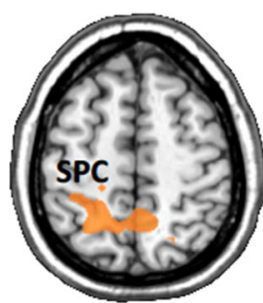

Fig. 5 Between-group (experimental > control) SPM t-maps during hand movement execution during Pre2 with respect to Post (Pre2 > Post). A significant decrease of BOLD activity was observed in the superior
62

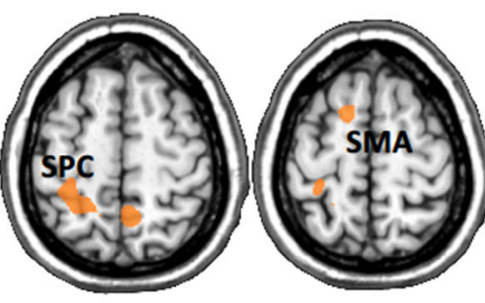

parietal cortex and supplementary motor area, in the contralesional hemisphere. Axial images are oriented according to neurological convention. LS = lesional side 
Fig. 6 Top, between-group differences (experimental > control) of contralesional SMA functional connectivity with whole brain (weighted seed-tovoxel analysis) during paretic hand movement after BMI training (Pre1 > Post). The contralesional SMA showed decreased positive correlation (warm colors) with contralesional cerebellum and contralesional premotor cortex, and decreased negative correlation (cool colors), with the ipsilesional premotor/ motor cortex, the putamen, and the ventromedial prefrontal cortex. Bottom, between-group differences (experimental > control) of contralesional M1 functional connectivity with whole brain (weighted seed-tovoxel analysis) during paretic hand movement after BMI training (Pre1 > Post). The contralesional M1 showed decreased negative correlation with ipsilesional premotor cortex, primary motor cortex, cingulate cortex, and parietal cortex (cool colors) and decreased positive correlation (warm colors) with contra- and ipsilesional cerebellum. Axial images are oriented according to neurological convention. $\mathrm{LS}=$ lesional side

SMA

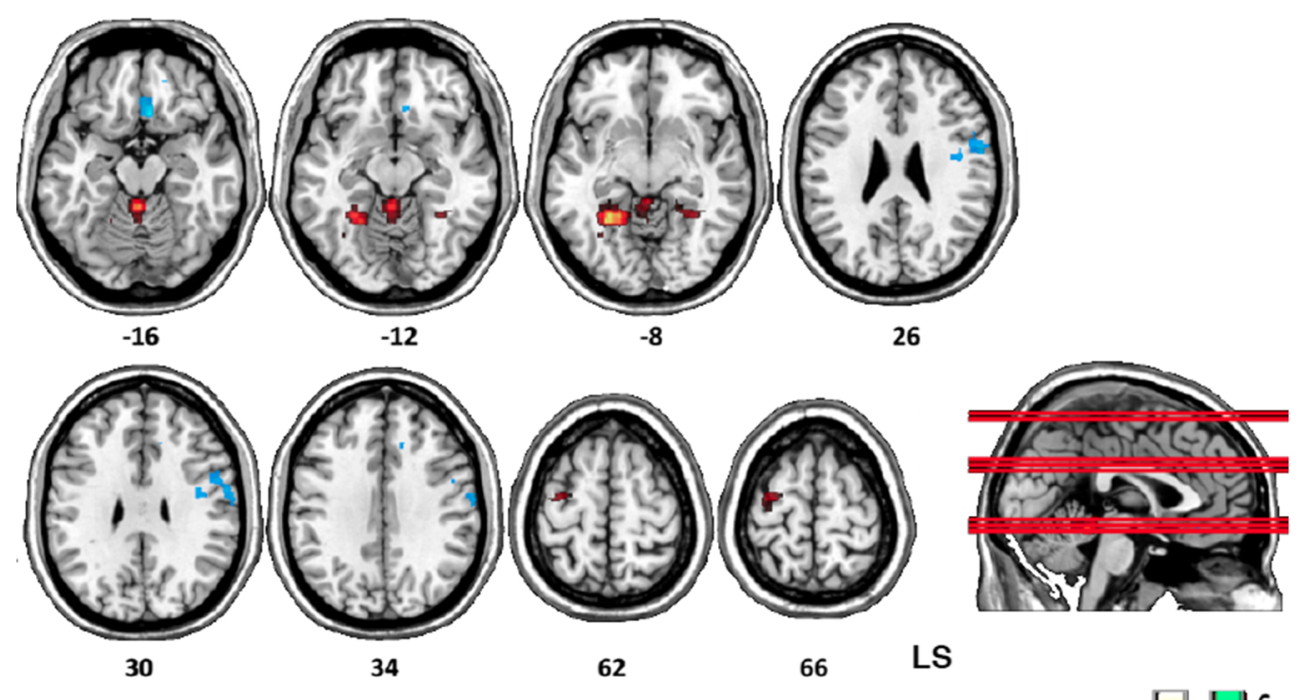

M1

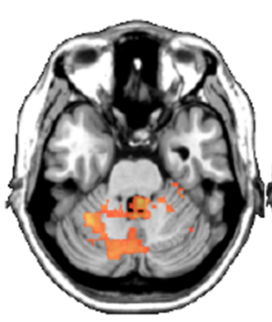

$-28$

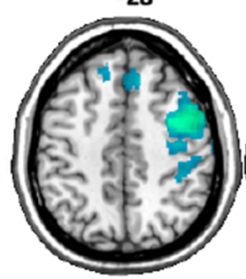

46

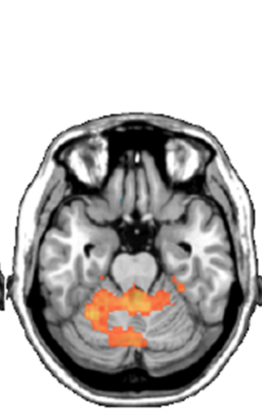

$-24$

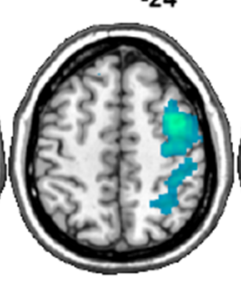

50

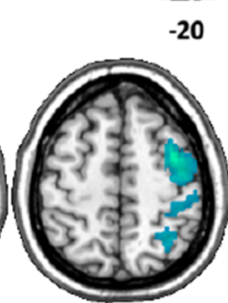

54

$-20$

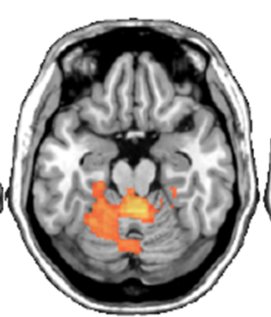

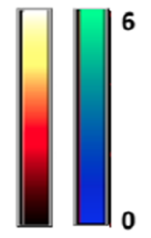

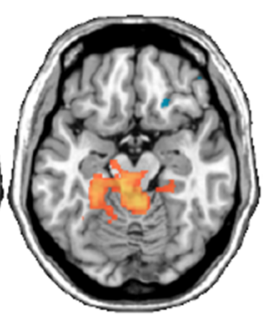

$-16$

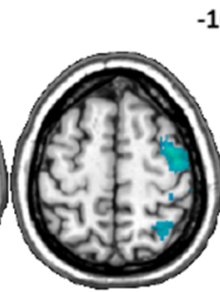

58

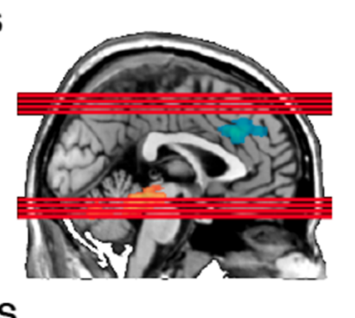

LS to negative (Post), whereas decreased negative coupling corresponded to changes of correlation and from negative (Pre2) to positive (Post). No significant between-group correlation differences were observed considering the contralesional SPC as seed. The comparison between Pre1 and Pre2 sessions did not reveal any significant difference for any of the selected seed.

\section{Structure-Function Relationship}

Correlation analysis between LI and FA changes explored the relationship between structural and functional changes after BMI training. A positive correlation was measured between Post-Pre2 LI changes and Post-Pre2 FA changes in the splenium (Pearson's $r=0.51, \mathrm{df}=13, p<0.05,1$-tailed) and in the contralesional posterior thalamic radiation (Pearson's $r=0.56, \mathrm{df}=13, p<0.05,1$-tailed).

\section{Discussion}

In this study, we investigated structural and functional motor network reorganization in severely impaired chronic stroke patients that underwent EEG-BMI-based treatment reinforcing sensorimotor contingency of ipsilesional motor commands. SVM-based classification of DTI metrics revealed significant white matter fractional anisotropy changes in the experimental group only. Specifically, FA decreased in several subcortical contralesional sensorimotor pathways and in the corpus callosum. Functional data analyses showed decreased 
BOLD activity in the contralesional supplementary motor area, superior parietal cortex, in the experimental group with respect to the control group. Furthermore, functional connectivity analyses corroborated these results by revealing decreased negative interhemispheric coupling between contralesional and ipsilesional sensorimotor regions, and decreased positive intrahemispheric coupling among contralesional sensorimotor regions, during paretic hand movement after training.

Whereas fMRI results are in line with longitudinal studies showing shifted activity towards the ipsilesional/contralateral sensorimotor regions during successful recovery after stroke $[27,70-72]$, the reduction of FA of white matter fiber tracts in the unaffected hemisphere as well as in the body and splenium of the corpus callosum is noteworthy. Most previous studies on recovery after stroke reported higher FA in contralesional white matter structures during acute to sub-acute phases of subcortical infarction [73], as well as in chronic patients, correlating with motor skill recovery [74], and thus proposing FA increase as index of a possible compensatory contribution of the ipsilateral/contralesional motor network [75]. Accordingly, reduced contralesional CST integrity, assessed with FA metrics as well as with motor evoked responses to TMS, was observed in chronic patients with poorer motor recovery as compared with patients with better motor skills and with control subjects $[19,74,76]$. A cross-sectional study investigating the relationship between FA and motor skills of the affected hand in chronic stroke demonstrated that patients with poor motor skills had reduced FA of bilateral corticospinal tract compared with healthy controls, whereas patients with better motor skills had increased FA of bilateral CST compared with controls [74]. However, in this case, it was unclear whether FA changes were due to the effects of ischemic damage, to secondary degeneration, or to positive remodeling of the tracts during recovery [77]. On the other hand, lower FA can also result from (re-)learning-related white matter modifications such as axon diameter increase and increased number of crossing fibers [78].

At present, the interpretation of FA variations remains unclear mainly because of its susceptibility to a combination of different tissue properties such as fiber arrangements, degree of myelination, and axonal integrity. Some evidences indicate that the main factor influencing FA might be axonal membranes more than myelin, although the latter can also participate in its modulation [79]. In our study, although clear inferences about the specific microstructural changes cannot be drawn, we observed that FA decrease was associated with a tendential increment of radial diffusivity. However, heterogeneity of $\mathrm{AD}$ and $\mathrm{RD}$ variations among patients suggest that multiple factors might have differentially contributed to the observed FA decrease in the white matter regions [44].

Nevertheless, it is conceivable that re-learning of motor acts through a treatment stimulating ipsilesional motor activity reduced the recruitment of contralesional hemisphere that in turn resulted in structural motor network modifications. For instance, a previous study reported contralesional decrease and ipsilesional increase of gray matter density in the primary sensorimotor cortex in chronic stroke patients showing shift of cortical activity from the healthy to the paretic hemisphere after intervention [80]. In line with our observation, a longitudinal study examining white matter changes in the contralesional hemisphere of chronic patients who recovered well from ischemic stroke observed a significant decrease of FA in the contralesional mirror region [81], suggesting that a possible degeneration of interhemispheric connections between homologous areas might facilitate recovery after stroke [23]. FA reduction in contralesional white matter regions was also observed in chronic stroke patients with Broca's aphasia showing improvements in speech production after intensive intonation-based speech therapy [82]. In addition, reduced FA and increased RD were measured in the corpus callosum of motor-impaired patients following amputation [83]. Interestingly, we observed a correlation between FA decrease in the splenium, and in the contralesional posterior thalamic radiation, the former encompassing somatosensory interhemispheric connections and the latter including thalamo-cortical fibers projecting to the sensorimotor cortex, with motor improvement and with increased lateralization towards the ipsilesional hemisphere. The concurrent FA decrease in the corpus callosum, and in the contralesional white matter fiber tracts, with decreased contralesional parietal activity might further indicate reduced contribution of the unaffected hemisphere.

CST damage after stroke often implies that proprioceptive input of the affected hand is additionally processed by contralesional somatosensory and parietal cortex, and in some cases ipsilesional somatosensory regions are completely inactive [84]. A meta-analysis of neuroimaging studies of hand motor task execution in stroke patients consistently showed the engagement of several contralesional areas such as M1, premotor, and supplementary motor areas [85]. Studies in macaques revealed concurrent primary motor and cerebellar activity in the contralesional hemisphere after corticospinal lesion in macaques and that pharmacological inactivation of contralateral M1 deteriorated hand motor functions only during early period of recovery and not during later stages, suggesting time-dependent adaptive contribution of the contralesional motor regions [72]. Accordingly, several studies confirmed this early observation showing reduced contralesional activations in successful motor recovery [86].

Increased exploitation of preserved ipsilesional motor connections and reduced engagement of the unaffected sensorimotor cortex might be also consistent with the observed reduction of FA in the contralesional posterior limb of the internal capsule, a portion of the corticospinal tract conducting motor efferences, as well as in the superior corona radiata, that 
includes somatomotor cortico-thalamic and cortico-pontine projections. The posterior limb of the internal capsule does not only contain corticofugal projections but also ascending axons to somatosensory cortex, and after stroke, the contralateral ascending sensory pathway might be partially or entirely disrupted. It is thus plausible that contralesional FA decrease might be also ascribable to the reduced exploitation of ipsilateral pathways for sensory afferences after training.

To date, the functional relevance of unaffected sensorimotor areas is still largely debated. The controversial evidences on the role of the contralesional hemisphere after stroke generated 2 alternative models describing possible neural mechanisms after brain damage: the "vicariation model" and the "interhemispheric competition model" [87]. The former model assumes that residual motor networks replace the activity in affected regions, and activity in the unaffected hemisphere would compensate for damaged or disconnected regions [88, $89]$ and contribute to recovery. On the other hand, the involvement of the non-lesioned hemisphere may be maladaptive and prevent motor functional improvement, in particular in the chronic stage [90-92]. Increased contralesional sensorimotor cortex responses have been observed in patients with poor functional outcomes [93, 94]. Moreover, an association between increased FA of the contralesional corticospinal tract and poor motor function has been also reported [10], indicating maladaptive structural reorganization of the contralesional hemisphere in non-treated stroke patients.

According to the "interhemispheric competition model," after brain infarct, the balanced inhibition between hemispheres is disrupted so increased inhibition of the damaged hemisphere from the healthy hemisphere is induced by decreased inhibition in the opposite direction. This reciprocal mechanism known as interhemispheric inhibition (IHI) in healthy subjects prevents the occurrence of mirror activity in the ipsilateral primary motor cortex $[95,96]$, whereas in patients after stroke, it would inhibit appropriate motor execution $[23,97,98]$. The IHI is mostly mediated by the corpus callosum $[66,99,100]$ and although M1 plays a major role, the interhemispheric inhibitory network includes secondary sensorimotor regions such as the SMA and pre-SMA [64, $101]$, the dorsal premotor cortex $[102,103]$, and the somatosensory cortex [104]. In addition, the cerebellum can also influence the inhibitory motor network at the cortical level [105]. On the other hand, it has been suggested that after stroke, mirror movements might be mediated by subcortical instead of cortical networks, suggesting involvement of rubrospinal and reticulospinal pathways [106, 107].

Previous functional connectivity studies in stroke patients reported that movements of the paretic hand are characterized by abnormal interhemispheric interactions from contralesional to ipsilesional primary motor cortex and SMA $[22,64]$. Notably, the degree of motor impairment was associated with the level of inhibitory influence from the contralesional M1 to the ipsilesional M1 [64]. Our functional connectivity analyses revealed large interhemispheric and intrahemispheric interactions in the contralesional hemisphere before training. Contralesional M1 positively correlated with bilateral cerebellum and negatively with ipsilesional premotor, primary motor, and parietal cortices; contralesional SMA positively correlated with contralesional and ipsilateral cerebellum and contralesional premotor cortex, and negatively with ipsilesional sensorimotor regions. These results confirm previous observations of bilateral sensorimotor region recruitment and increased intrahemispheric coupling among contralesional motor network nodes [108]. In particular, they are in line with studies indicating that ipsilateral premotor cortex, in addition to M1, would support hand movement after stroke $[88,109]$.

In addition, we observed positive cerebellar coupling with contralesional M1 and SMA before training. Increasing evidence indicates that the cerebellum, a region essential for motor coordination and learning with no direct projections to peripheral motor neurons, might also participate to stroke recovery $[110,111]$. Though, there is still no consensus on its role: on the one hand, some studies showed motor function enhancement after cerebellar stimulation [110], suggesting a beneficial role for motor impairment compensation after stroke, and on the other hand, other studies suggest a possible association with interhemispheric inhibition [105].

Remarkably, after training, in the experimental group, as compared with the control group, both contralesional M1 and SMA showed reduced positive coupling with contralesional sensorimotor regions and reduced negative coupling with ipsilesional sensorimotor network. The reduced contralesional intrahemispheric connectivity involving M1 and SMA, critical nodes of the interhemispheric inhibitory network along with decreased negative interhemispheric sensorimotor interactions, might here suggest a detrimental role of the unaffected hemisphere, potentially exerted through abnormal IHI.

Previous studies showed a positive correlation between microstructural integrity of the corpus callosum (FA-based) and strength of interhemispheric inhibition in adults $[100,112]$ and in the course of motor development [113]. In particular, it has been shown that increased FA predicts interhemispheric inhibitory capacity [69]. In our study, the measured FA decrease in the body and splenium of the corpus callosum, including premotor and somatosensory interhemispheric fibers, and in the posterior limb of the internal capsule and superior corona radiata in the contralesional hemisphere, which conduct hemisphere-crossing tracts mostly from pre- and supplementary motor regions, might result from reduced contralesional activity, and potentially decreased maladaptive transcallosal communication. Furthermore, the decreased negative coupling of M1 with the cerebellum along with reduced FA in the contralateral superior corona radiata, a region that includes cortico-pontine fibers interconnecting the cerebral cortex with the cerebellum 
via pontocerebellar fibers, might indicate remodeling of the inhibitory motor network at different brain levels mediating motor execution and coordination improvement.

In short, independent of the functional relevance of the contralesional activity, either beneficial or detrimental, altogether, our functional data indicate a reduced contribution of the ipsilateral hemisphere as well as modifications of interhemispheric interactions after EEG-BMI training. As transcallosal pathways contain both inhibitory and facilitatory fibers with a number of synapses, it is not possible here to clearly interpret the nature of interhemispheric communications $[69,114]$, and further studies addressing the effects of BMI-based intervention on interhemispheric interactions should be specifically tailored.

Overall, although sample size is limited, our findings indicate that BMI training reinforcing ipsilesional brain activity and enhancing proprioceptive function of the affected hand elicits intra- and inter-hemispheric reorganization of somatosensory and motor-assemblies as well as afferent and efferent connection-related motor circuits [115] that support the partial re-establishment of the original neurophysiology of the motor system. Notably, whereas functional reorganization involved both hemispheres, white matter FA changes were mainly related to interhemispheric connections and the contralesional hemisphere. This observation suggests that recovery in severely impaired chronic stroke patients may be possibly achieved through reorganization of the unaffected hemisphere and exploitation of the remaining preserved motor circuits in the affected hemisphere.

The chronic stage has long been assumed a phase in which the adaptive processes of regeneration cease completely and, consequently, the range of treatment options has been very narrow and the focus of the clinical community has centered on palliative and assistive interventions. On the contrary, our results highlight the significant role of non-invasive BMI approach for stimulating preserved brain systems, otherwise not reachable in patients with severe motor network disruptions that can elicit functionally adaptive mechanisms fostering motor recovery.

Acknowledgments This work was supported by the German Federal Ministry of Education and Research (BMBF, Förderzeichen 01GQ0831); Deutsche Forschungsgemeinschaft (DFG) Koselleck (BI 195/58-1); European Research Council (ERC 227632); European Union FP7-ICT-2009-231724 - HUMOR: Human Behavioral Modeling for Enhancing Learning by Optimizing Human-Robot Interaction, FP7ICT-2009-247935 - BETTER: BNCI-driven Robotic Physical Therapies in Stroke Rehabilitation of Gait Disorders; Italian Ministry of Health GR2009-159190; BW-Stiftung (ROB-1); Werner Reichardt Centre for Integrative Neuroscience (CIN). The authors would like to thank Balint Varkuti, Leonhard Läer, Pavel Terekhin, Nicolas Lindau, and Bjorn Schiffler for their support.

Required Author Forms Disclosure forms provided by the authors are available with the online version of this article.

\section{Compliance with Ethical Standards}

Informed consent, including informed consent to publish identifying information/images in an online open-access publication, was obtained from all patients involved. The study was approved by the ethics committee of the Faculty of Medicine of the University of Tübingen. The methods carried out in this work are in accordance with the approved guidelines and regulations.

Competing Interests The authors declare that they have no competing interests.

\section{References}

1. Hochberg LR, Bacher D, Jarosiewicz B et al. Reach and grasp by people with tetraplegia using a neurally controlled robotic arm. Nature, 485(7398), 372-375 (2012).

2. Hochberg LR, Serruya MD, Friehs GM et al. Neuronal ensemble control of prosthetic devices by a human with tetraplegia. Nature, 442(7099), 164-171 (2006).

3. Collinger JL, Wodlinger B, Downey JE et al. High-performance neuroprosthetic control by an individual with tetraplegia. Lancet, 381(9866), 557-564 (2013).

4. Buch E, Weber C, Cohen LG et al. Think to move: a neuromagnetic brain-computer interface (BCI) system for chronic stroke. Stroke, 39(3), 910-917 (2008).

5. Bundy DT, Wronkiewicz M, Sharma M, Moran DW, Corbetta M, Leuthardt EC. Using ipsilateral motor signals in the unaffected cerebral hemisphere as a signal platform for brain-computer interfaces in hemiplegic stroke survivors. J Neural Eng, 9(3), 036011 (2012).

6. Ang KK, Guan C, Chua KS et al. Clinical study of neurorehabilitation in stroke using EEG-based motor imagery brain-computer interface with robotic feedback. Conf Proc IEEE Eng Med Biol Soc, 2010, 5549-5552 (2010).

7. Shindo K, Kawashima K, Ushiba J et al. Effects of neurofeedback training with an electroencephalogram-based brain-computer interface for hand paralysis in patients with chronic stroke: a preliminary case series study. J Rehabil Med, 43(10), 951-957 (2011).

8. Young BM, Nigogosyan Z, Remsik A et al. Changes in functional connectivity correlate with behavioral gains in stroke patients after therapy using a brain-computer interface device. Front Neuroeng, 7, 25 (2014)

9. Young BM, Nigogosyan Z, Walton LM et al. Changes in functional brain organization and behavioral correlations after rehabilitative therapy using a brain-computer interface. Front Neuroeng, 7, 26 (2014).

10. Young BM, Stamm JM, Song J et al. Brain-Computer Interface Training after Stroke Affects Patterns of Brain-Behavior Relationships in Corticospinal Motor Fibers. Front Hum Neurosci, 10, 457 (2016).

11. Birbaumer N, Cohen LG. Brain-computer interfaces: communication and restoration of movement in paralysis. J Physiol, 579(Pt 3), 621-636 (2007).

12. Caria A, Weber C, Brotz D et al. Chronic stroke recovery after combined BCI training and physiotherapy: a case report. Psychophysiology, 48(4), 578-582 (2011).

13. Varkuti B, Guan C, Pan Y et al. Resting state changes in functional connectivity correlate with movement recovery for $\mathrm{BCI}$ and robotassisted upper-extremity training after stroke. Neurorehabil Neural Repair, 27(1), 53-62 (2013).

14. Daly JJ, Wolpaw JR. Brain-computer interfaces in neurological rehabilitation. Lancet Neurol, 7(11), 1032-1043 (2008). 
15. Ramos-Murguialday A, Broetz D, Rea M et al. Brain-machine interface in chronic stroke rehabilitation: a controlled study. Ann Neurol, 74(1), 100-108 (2013).

16. Krakauer JW. Motor learning: its relevance to stroke recovery and neurorehabilitation. Curr Opin Neurol, 19(1), 84-90 (2006).

17. Pomeroy V, Aglioti SM, Mark VW et al. Neurological principles and rehabilitation of action disorders: rehabilitation interventions. Neurorehabil Neural Repair, 25(5 Suppl), 33S-43S (2011).

18. Ward NS, Cohen LG. Mechanisms underlying recovery of motor function after stroke. Arch Neurol, 61(12), 1844-1848 (2004).

19. Stinear CM, Barber PA, Smale PR, Coxon JP, Fleming MK, Byblow WD. Functional potential in chronic stroke patients depends on corticospinal tract integrity. Brain, 130(Pt 1), 170-180 (2007)

20. Liepert J, Hamzei F, Weiller C. Motor cortex disinhibition of the unaffected hemisphere after acute stroke. Muscle Nerve, 23(11), 1761-1763 (2000)

21. Hummel FC, Cohen LG. Non-invasive brain stimulation: a new strategy to improve neurorehabilitation after stroke? Lancet Neurol, 5(8), 708-712 (2006).

22. Rehme AK, Eickhoff SB, Wang LE, Fink GR, Grefkes C. Dynamic causal modeling of cortical activity from the acute to the chronic stage after stroke. Neuroimage, 55(3), 1147-1158 (2011).

23. Murase N, Duque J, Mazzocchio R, Cohen LG. Influence of interhemispheric interactions on motor function in chronic stroke. Ann Neurol, 55(3), 400-409 (2004).

24. Carey JR, Kimberley TJ, Lewis SM et al. Analysis of fMRI and finger tracking training in subjects with chronic stroke. Brain, 125(Pt 4), 773-788 (2002).

25. Carey LM, Abbott DF, Egan GF, Bernhardt J, Donnan GA. Motor impairment and recovery in the upper limb after stroke: behavioral and neuroanatomical correlates. Stroke, 36(3), 625-629 (2005).

26. Rossini PM, Dal Forno G. Neuronal post-stroke plasticity in the adult. Restor Neurol Neurosci, 22(3-5), 193-206 (2004).

27. Ward NS, Brown MM, Thompson AJ, Frackowiak RS. Neural correlates of motor recovery after stroke: a longitudinal fMRI study. Brain, 126(Pt 11), 2476-2496 (2003).

28. Ward NS, Brown MM, Thompson AJ, Frackowiak RS. Neural correlates of outcome after stroke: a cross-sectional fMRI study. Brain, 126(Pt 6), 1430-1448 (2003).

29. Loubinoux I, Carel C, Pariente J et al. Correlation between cerebral reorganization and motor recovery after subcortical infarcts. Neuroimage, 20(4), 2166-2180 (2003).

30. Broetz D, Braun C, Weber C, Soekadar SR, Caria A, Birbaumer $\mathrm{N}$. Combination of brain-computer interface training and goaldirected physical therapy in chronic stroke: a case report. Neurorehabil Neural Repair, 24(7), 674-679 (2010).

31. Song J, Nair VA, Young BM et al. DTI measures track and predict motor function outcomes in stroke rehabilitation utilizing BCI technology. Front Hum Neurosci, 9, 195 (2015).

32. Song J, Young BM, Nigogosyan $\mathrm{Z}$ et al. Characterizing relationships of DTI, fMRI, and motor recovery in stroke rehabilitation utilizing brain-computer interface technology. Front Neuroeng, 7, 31 (2014).

33. Marins T, Rodrigues EC, Bortolini T, Melo B, Moll J, Tovar-Moll F. Structural and functional connectivity changes in response to short-term neurofeedback training with motor imagery. Neuroimage, 194, 283-290 (2019).

34. Tyc F, Boyadjian A. Plasticity of motor cortex induced by coordination and training. Clin Neurophysiol, 122(1), 153-162 (2011).

35. Uswatte G, Taub E, Morris D, Vignolo M, McCulloch K. Reliability and validity of the upper-extremity Motor Activity Log-14 for measuring real-world arm use. Stroke, 36(11), 2493 2496 (2005)
36. Hurn J, Kneebone I, Cropley M. Goal setting as an outcome measure: A systematic review. Clin Rehabil, 20(9), 756-772 (2006).

37. Platz T, Pinkowski C, van Wijck F, Kim IH, di Bella P, Johnson G. Reliability and validity of arm function assessment with standardized guidelines for the Fugl-Meyer Test, Action Research Arm Test and Box and Block Test: a multicentre study. Clin Rehabil, 19(4), 404-411 (2005).

38. Alexander AL, Lee JE, Lazar M, Field AS. Diffusion tensor imaging of the brain. Neurotherapeutics, 4(3), 316-329 (2007).

39. Le Bihan D. Looking into the functional architecture of the brain with diffusion MRI. Nat Rev Neurosci, 4(6), 469-480 (2003).

40. Zatorre RJ, Fields RD, Johansen-Berg H. Plasticity in gray and white: neuroimaging changes in brain structure during learning. Nat Neurosci, 15(4), 528-536 (2012).

41. Puig J, Blasco G, Schlaug G et al. Diffusion tensor imaging as a prognostic biomarker for motor recovery and rehabilitation after stroke. Neuroradiology, 59(4), 343-351 (2017).

42. Bennett IJ, Madden DJ, Vaidya CJ, Howard DV, Howard JH, Jr. Age-related differences in multiple measures of white matter integrity: A diffusion tensor imaging study of healthy aging. Hum Brain Mapp, 31(3), 378-390 (2010).

43. Winklewski PJ, Sabisz A, Naumczyk P, Jodzio K, Szurowska E, Szarmach A. Understanding the Physiopathology Behind Axial and Radial Diffusivity Changes-What Do We Know? Front Neurol, 9, 92 (2018).

44. Song SK, Yoshino J, Le TQ et al. Demyelination increases radial diffusivity in corpus callosum of mouse brain. Neuroimage, 26(1), 132-140 (2005).

45. Mori S, Oishi K, Jiang H et al. Stereotaxic white matter atlas based on diffusion tensor imaging in an ICBM template. Neuroimage, 40(2), 570-582 (2008).

46. Smola AJ, Scholkopf B. A tutorial on support vector regression. Stat Comput, 14(3), 199-222 (2004).

47. Yourganov G, Fridriksson J, Rorden C, Gleichgerrcht E, Bonilha L. Multivariate Connectome-Based Symptom Mapping in PostStroke Patients: Networks Supporting Language and Speech. J Neurosci, 36(25), 6668-6679 (2016).

48. Cuingnet R, Rosso C, Chupin $\mathrm{M}$ et al. Spatial regularization of SVM for the detection of diffusion alterations associated with stroke outcome. Med Image Anal, 15(5), 729-737 (2011).

49. Cuingnet R, Gerardin E, Tessieras J et al. Automatic classification of patients with Alzheimer's disease from structural MRI: a comparison of ten methods using the ADNI database. Neuroimage, 56(2), 766-781 (2011).

50. Lao Z, Shen D, Xue Z, Karacali B, Resnick SM, Davatzikos C. Morphological classification of brains via high-dimensional shape transformations and machine learning methods. Neuroimage, 21(1), 46-57 (2004).

51. Kloppel S, Stonnington CM, Chu C et al. Automatic classification of MR scans in Alzheimer's disease. Brain, 131(Pt 3), 681-689 (2008)

52. Vemuri P, Gunter JL, Senjem ML et al. Alzheimer's disease diagnosis in individual subjects using structural MR images: validation studies. Neuroimage, 39(3), 1186-1197 (2008).

53. Kriegeskorte N, Goebel R, Bandettini P. Information-based functional brain mapping. Proc Natl Acad Sci U S A, 103(10), 3863 3868 (2006).

54. Dalboni da Rocha JL, Coutinho G, Bramati I, Moll FT, Sitaram R. Multilevel diffusion tensor imaging classification technique for characterizing neurobehavioral disorders. Brain Imaging Behav, 1-12. https://doi.org/10.1007/s11682-018-0002-2 (2018).

55. Zurita M, Montalba $\mathrm{C}$, Labbe $\mathrm{T}$ et al. Characterization of relapsing-remitting multiple sclerosis patients using support vector machine classifications of functional and diffusion MRI data. NeuroImage Clin, 20, 724-730 (2018). 
56. Andersen SM, Rapcsak SZ, Beeson PM. Cost function masking during normalization of brains with focal lesions: still a necessity? Neuroimage, 53(1), 78-84 (2010).

57. Lieberman MD, Cunningham WA. Type I and Type II error concerns in fMRI research: re-balancing the scale. Soc Cogn Affect Neurosci, 4(4), 423-428 (2009).

58. Slotnick SD. Cluster success: fMRI inferences for spatial extent have acceptable false-positive rates. Cogn Neurosci, 8(3), 150 155 (2017)

59. Cunningham WA, Koscik TR. Balancing Type I and Type II error concerns in fMRI through compartmentalized analysis. Cogn Neurosci, 8(3), 147-149 (2017).

60. Smith SM, Nichols TE. Threshold-free cluster enhancement: addressing problems of smoothing, threshold dependence and localisation in cluster inference. Neuroimage, 44(1), 83-98 (2009).

61. Spisak T, Spisak Z, Zunhammer M et al. Probabilistic TFCE: A generalized combination of cluster size and voxel intensity to increase statistical power. Neuroimage, 185, 12-26 (2018).

62. Wilke M, Lidzba K. LI-tool: a new toolbox to assess lateralization in functional MR-data. J Neurosci Methods, 163(1), 128-136 (2007).

63. Whitfield-Gabrieli S, Nieto-Castanon A. Conn: a functional connectivity toolbox for correlated and anticorrelated brain networks. Brain Connect, 2(3), 125-141 (2012).

64. Grefkes C, Nowak DA, Eickhoff SB et al. Cortical connectivity after subcortical stroke assessed with functional magnetic resonance imaging. Ann Neurol, 63(2), 236-246 (2008).

65. Grefkes C, Fink GR. Reorganization of cerebral networks after stroke: new insights from neuroimaging with connectivity approaches. Brain, 134(Pt 5), 1264-1276 (2011).

66. Palmer LM, Schulz JM, Murphy SC, Ledergerber D, Murayama $\mathrm{M}$, Larkum ME. The cellular basis of GABA(B)-mediated interhemispheric inhibition. Science, 335(6071), 989-993 (2012).

67. Ramos-Murguialday A, Curado MR, Broetz D et al. BrainMachine Interface in Chronic Stroke: Randomized Trial LongTerm Follow-up. Neurorehabil Neural Repair, 33(3), 188-198 (2019).

68. Hofer S, Frahm J. Topography of the human corpus callosum revisited-comprehensive fiber tractography using diffusion tensor magnetic resonance imaging. Neuroimage, 32(3), 989-994 (2006)

69. Fling BW, Benson BL, Seidler RD. Transcallosal sensorimotor fiber tract structure-function relationships. Hum Brain Mapp, 34(2), 384-395 (2013).

70. Calautti C, Leroy F, Guincestre JY, Marie RM, Baron JC. Sequential activation brain mapping after subcortical stroke: changes in hemispheric balance and recovery. Neuroreport, 12(18), 3883-3886 (2001).

71. Marshall RS, Perera GM, Lazar RM, Krakauer JW, Constantine RC, DeLaPaz RL. Evolution of cortical activation during recovery from corticospinal tract infarction. Stroke, 31(3), 656-661 (2000).

72. Nishimura Y, Onoe H, Morichika Y, Perfiliev S, Tsukada H, Isa T. Time-dependent central compensatory mechanisms of finger dexterity after spinal cord injury. Science, 318(5853), 1150-1155 (2007).

73. Liu G, Dang C, Chen X et al. Structural remodeling of white matter in the contralesional hemisphere is correlated with early motor recovery in patients with subcortical infarction. Restor Neurol Neurosci, 33(3), 309-319 (2015).

74. Schaechter JD, Fricker ZP, Perdue KL et al. Microstructural status of ipsilesional and contralesional corticospinal tract correlates with motor skill in chronic stroke patients. Hum Brain Mapp, 30(11), 3461-3474 (2009).
75. Koch P, Schulz R, Hummel FC. Structural connectivity analyses in motor recovery research after stroke. Ann Clin Transl Neurol, 3(3), 233-244 (2016).

76. Jang SH, Cho SH, Kim YH et al. Diffusion anisotrophy in the early stages of stroke can predict motor outcome. Restor Neurol Neurosci, 23(1), 11-17 (2005).

77. Johansen-Berg H, Scholz J, Stagg CJ. Relevance of structural brain connectivity to learning and recovery from stroke. Front Syst Neurosci, 4, 146 (2010).

78. Taubert M, Draganski B, Anwander A et al. Dynamic properties of human brain structure: learning-related changes in cortical areas and associated fiber connections. J Neurosci, 30(35), 11670 11677 (2010).

79. Beaulieu C. Chapter 8 - The Biological Basis of Diffusion Anisotropy. In: Diffusion MRI (2nd Edition). (Academic Press, San Diego, 2014) 155-183.

80. Wilkins KB, Owen M, Ingo C, Carmona C, Dewald JPA, Yao J. Neural Plasticity in Moderate to Severe Chronic Stroke Following a Device-Assisted Task-Specific Arm/Hand Intervention. Front Neurol, 8, 284 (2017).

81. Granziera C, Ay H, Koniak SP, Krueger G, Sorensen AG. Diffusion tensor imaging shows structural remodeling of stroke mirror region: results from a pilot study. Eur Neurol, 67(6), 370 376 (2012).

82. Wan CY, Zheng X, Marchina S, Norton A, Schlaug G. Intensive therapy induces contralateral white matter changes in chronic stroke patients with Broca's aphasia. Brain Lang, 136, 1-7 (2014).

83. $\mathrm{Li} \mathrm{Z}, \mathrm{Li} \mathrm{C}, \mathrm{Fan} \mathrm{L}$ et al. Altered microstructure rather than morphology in the corpus callosum after lower limb amputation. Sci Rep, 7, 44780 (2017).

84. Jang SH. Contra-lesional somatosensory cortex activity and somatosensory recovery in two stroke patients. J Rehabil Med, 43(3), 268-270 (2011).

85. Rehme AK, Eickhoff SB, Rottschy C, Fink GR, Grefkes C. Activation likelihood estimation meta-analysis of motor-related neural activity after stroke. Neuroimage, 59(3), 2771-2782 (2012)

86. Grefkes C, Ward NS. Cortical reorganization after stroke: how much and how functional? Neuroscientist, 20(1), 56-70 (2014).

87. Di Pino G, Pellegrino G, Assenza G et al. Modulation of brain plasticity in stroke: a novel model for neurorehabilitation. Nat Rev Neurol, 10(10), 597-608 (2014).

88. Johansen-Berg H, Rushworth MF, Bogdanovic MD, Kischka U, Wimalaratna S, Matthews PM. The role of ipsilateral premotor cortex in hand movement after stroke. Proc Natl Acad Sci U S A, 99(22), 14518-14523 (2002).

89. Schaechter JD, Perdue KL. Enhanced cortical activation in the contralesional hemisphere of chronic stroke patients in response to motor skill challenge. Cereb Cortex, 18(3), 638-647 (2008).

90. Eyre JA. Corticospinal tract development and its plasticity after perinatal injury. Neurosci Biobehav Rev, 31(8), 1136-1149 (2007).

91. Fregni F, Pascual-Leone A. Hand motor recovery after stroke: tuning the orchestra to improve hand motor function. Cogn Behav Neurol, 19(1), 21-33 (2006).

92. Nowak DA, Grefkes C, Dafotakis M et al. Effects of lowfrequency repetitive transcranial magnetic stimulation of the contralesional primary motor cortex on movement kinematics and neural activity in subcortical stroke. Arch Neurol, 65(6), 741-747 (2008)

93. Schwerin S, Dewald JP, Haztl M, Jovanovich S, Nickeas M, MacKinnon C. Ipsilateral versus contralateral cortical motor projections to a shoulder adductor in chronic hemiparetic stroke: implications for the expression of arm synergies. Exp Brain Res, 185(3), 509-519 (2008). 
94. Ward NS, Newton JM, Swayne OB et al. Motor system activation after subcortical stroke depends on corticospinal system integrity. Brain, 129(Pt 3), 809-819 (2006).

95. Daskalakis ZJ, Christensen BK, Fitzgerald PB, Roshan L, Chen R. The mechanisms of interhemispheric inhibition in the human motor cortex. J Physiol, 543(Pt 1), 317-326 (2002).

96. Duque J, Murase N, Celnik P et al. Intermanual Differences in movement-related interhemispheric inhibition. J Cogn Neurosci, 19(2), 204-213 (2007).

97. Duque J, Hummel F, Celnik P, Murase N, Mazzocchio R, Cohen LG. Transcallosal inhibition in chronic subcortical stroke. Neuroimage, 28(4), 940-946 (2005).

98. Avanzino L, Bassolino M, Pozzo T, Bove M. Use-dependent hemispheric balance. J Neurosci, 31(9), 3423-3428 (2011).

99. Avanzino L, Teo JT, Rothwell JC. Intracortical circuits modulate transcallosal inhibition in humans. J Physiol, 583(Pt 1), 99-114 (2007).

100. Wahl M, Lauterbach-Soon B, Hattingen E et al. Human motor corpus callosum: topography, somatotopy, and link between microstructure and function. J Neurosci, 27(45), 12132-12138 (2007).

101. Serrien DJ, Strens LH, Oliviero A, Brown P. Repetitive transcranial magnetic stimulation of the supplementary motor area (SMA) degrades bimanual movement control in humans. Neurosci Lett, 328(2), 89-92 (2002).

102. Giovannelli F, Borgheresi A, Balestrieri F et al. Role of the right dorsal premotor cortex in "physiological" mirror EMG activity Exp Brain Res, 175(4), 633-640 (2006).

103. van den Berg FE, Swinnen SP, Wenderoth N. Hemispheric asymmetries of the premotor cortex are task specific as revealed by disruptive TMS during bimanual versus unimanual movements. Cereb Cortex, 20(12), 2842-2851 (2010).

104. Ni Z, Gunraj C, Nelson AJ et al. Two phases of interhemispheric inhibition between motor related cortical areas and the primary motor cortex in human. Cereb Cortex, 19(7), 1654-1665 (2009).

105. Daskalakis ZJ, Paradiso GO, Christensen BK, Fitzgerald PB, Gunraj C, Chen R. Exploring the connectivity between the cerebellum and motor cortex in humans. J Physiol, 557(Pt 2), 689-700 (2004).
106. Ejaz N, Xu J, Branscheidt $\mathrm{M}$ et al. Evidence for a subcortical origin of mirror movements after stroke: a longitudinal study. Brain, 141(3), 837-847 (2018).

107. Calautti C, Jones PS, Naccarato $M$ et al. Further evidence for a non-cortical origin of mirror movements after stroke. Brain, 142(1), e1 (2019).

108. Lam TK, Dawson DR, Honjo K et al. Neural coupling between contralesional motor and frontoparietal networks correlates with motor ability in individuals with chronic stroke. J Neurol Sci, 384, 21-29 (2018).

109. Ludemann-Podubecka J, Bosl K, Nowak DA. Inhibition of the contralesional dorsal premotor cortex improves motor function of the affected hand following stroke. Eur J Neurol, 23(4), 823830 (2016).

110. Celnik P. Understanding and modulating motor learning with cerebellar stimulation. Cerebellum, 14(2), 171-174 (2015).

111. Spampinato DA, Block HJ, Celnik PA. Cerebellar-M1 Connectivity Changes Associated with Motor Learning Are Somatotopic Specific. J Neurosci, 37(9), 2377-2386 (2017).

112. Wahl M, Lauterbach-Soon B, Hattingen E, Hubers A, Ziemann U. Callosal anatomical and effective connectivity between primary motor cortices predicts visually cued bimanual temporal coordination performance. Brain Struct Funct, 221(7), 3427-3443 (2016).

113. Koerte I, Heinen F, Fuchs T et al. Anisotropy of callosal motor fibers in combination with transcranial magnetic stimulation in the course of motor development. Investig Radiol, 44(5), 279-284 (2009).

114. Takechi U, Matsunaga K, Nakanishi R et al. Longitudinal changes of motor cortical excitability and transcallosal inhibition after subcortical stroke. Clin Neurophysiol, 125(10), 2055-2069 (2014).

115. Zaaimi B, Edgley SA, Soteropoulos DS, Baker SN. Changes in descending motor pathway connectivity after corticospinal tract lesion in macaque monkey. Brain, 135(Pt 7), 2277-2289 (2012).

Publisher's Note Springer Nature remains neutral with regard to jurisdictional claims in published maps and institutional affiliations. 